\title{
The Role of Serum Chloride in Acute and Chronic Heart Failure: A Narrative Review
}

\author{
Frederick Berro Rivera $^{\mathrm{a}}$ Pia Alfonso ${ }^{\mathrm{b}}$ Jem Marie Golbin ${ }^{\mathrm{b}}$ Kevin Lo ${ }^{c}$ \\ Edgar Lerma $^{d}$ Annabelle Santos Volgman ${ }^{\mathrm{e}}$ Amir Kazory $^{f}$ \\ aDepartment of Medicine, Philippine General Hospital, University of the Philippines System, National Capital Region, \\ Manila, Philippines; b University of the Philippines System, National Capital Region, Manila, Philippines; ${ }^{\circ}$ Department \\ of Medicine, Einstein Medical Center, Philadelphia, PA, USA; ${ }^{d}$ Section of Nephrology, University of Illinois at Chicago \\ College of Medicine, Chicago, IL, USA; 'Division of Cardiology, Rush University Medical Center, Chicago, IL, USA; \\ fDivision of Nephrology, Hypertension, and Renal Transplantation, University of Florida, Gainesville, FL, USA
}

\section{Keywords}

Heart failure $\cdot$ Chloride $\cdot$ Cardiorenal syndrome $\cdot$ Congestion

\section{Abstract}

Clinical guidelines include diuretics for the treatment of heart failure (HF), not to decrease mortality but to decrease symptoms and hospitalizations. More attention has been paid to the worse outcomes, including mortality, associated with continual diuretic therapy due to hypochloremia. Studies have revealed a pivotal role for serum chloride in the pathophysiology of HF and is now a target of treatment to decrease mortality. The prognostic value of serum chloride in HF has been the subject of much attention. Mechanistically, the macula densa, a region in the renal juxtaglomerular apparatus, relies on chloride levels to sense salt and volume status. The recent discovery of with-no-lysine (K) (WNK) protein kinase as an intracellular chloride sensor sheds light on the possible reason of diuretic resistance in $\mathrm{HF}$. The action of chloride on WNKs results in the upregulation of the sodiumpotassium-chloride cotransporter and sodium-chloride cotransporter receptors, which could lead to increased electro- lyte and fluid reabsorption. Genetic studies have revealed that a variant of a voltage-sensitive chloride channel (CLCNKA) gene leads to almost a $50 \%$ decrease in current amplitude and function of the renal chloride channel. This variant increases the risk of HF. Several trials exploring the prognostic value of chloride in both acute and chronic HF have shown mostly positive results, some even suggesting a stronger role than sodium. However, so far, interventional trials exploring serum chloride as a therapeutic target have been largely inconclusive. This study is a review of the pathophysiologic effects of hypochloremia in $\mathrm{HF}$, the genetics of chloride channels, and clinical trials that are underway to investigate novel approaches to HF management.

(c) 2021 The Author(s)

Published by S. Karger AG, Basel

\section{Introduction}

Cardiovascular disease is the most commonly diagnosed medical condition among patients aged 65 years and above and is a major global problem with an estimated worldwide prevalence of 38 million [1]. Of those, there karger@karger.com www.karger.com/crm

Karger $\stackrel{\text { ' }}{5}$
(C) 2021 The Author(s)

Published by S. Karger AG, Basel

This is an Open Access article licensed under the Creative Common Attribution-NonCommercial-4.0 International License (CC BY-NC) (http://www.karger.com/Services/OpenAccessLicense), applicable to the online version of the article only. Usage and distribution for commercial purposes requires written permission.
Correspondence to:

Frederick Berro Rivera, frederick.berro.rivera@gmail.com 
were 6.2 million adults in the USA who have heart failure (HF), and this number is expected to rise in the future. The high morbidity and mortality rate associated with HF make it a challenging public concern with significant financial burden on healthcare [2].

Patients with HF frequently present with multiple electrolyte abnormalities, hyponatremia, hypokalemia, and hypochloremia being the most common. This is partly due to the reduced renal perfusion from the decreased cardiac output which stimulates the renin-angiotensinaldosterone system (RAAS) as well as arginine vasopressin and sympathetic nervous system (SNS) leading to fluid retention. While hyponatremia on admission has shown to be strongly associated with an increased risk of mortality, it is not considered a direct target for treatment since its correction does not seem to improve the outcomes [3]. On the other hand, serum chloride, an oftenoverlooked electrolyte, is the second most abundant electrolyte in the body, and emerging evidence points to its important role in this setting [4].

Clinical guidelines include diuretics for the treatment of HF, not to decrease mortality but to decrease symptoms and hospitalizations [5]. More attention has been paid to the worse outcomes, including mortality, associated with continual diuretic therapy due to hypochloremia. Studies have revealed a pivotal role for serum chloride in the pathophysiology of HF and is now a target of treatment to decrease mortality. The prognostic value of serum chloride in HF has been the subject of much attention [6]. In this review, we provide an overview of the currently available data on the physiologic role of serum chloride, its relevance in $\mathrm{HF}$ and congestive signs and symptoms, and its impact on the outcomes of these patients.

\section{Chloride and Volume Status}

Chloride concentration is regulated by the gastrointestinal tract and the kidneys [7]. As with most electrolytes, the proximal tubule is responsible for the bulk of the renal reabsorption of chloride. The thick ascending limb of the loop of Henle reabsorbs approximately $15-25 \%$ of filtered sodium chloride $(\mathrm{NaCl})$ through the $\mathrm{Na}+/ \mathrm{K}+/ 2 \mathrm{Cl}$ cotransporter (NKCC) [7]. The macula densa, a region in the renal juxtaglomerular apparatus, primarily relies on chloride levels in sensing salt load and volume status. If volume status is sensed as adequate, there is decreased reabsorption of $\mathrm{NaCl}$ in the proximal tubule and an increased concentration of $\mathrm{NaCl}$ at the macula densa. This in turn suppresses renin secretion, hence deactivating the RAAS. Interestingly, this neurohormonal effect occurs independent of sodium concentration as suggested by failure of renin suppression with administration of sodium bicarbonate compared to sodium chloride. The established role of chloride levels in regulation of renin secretion and volume status is the basis of the chloride theory for worsening $\mathrm{HF}$ [7].

\section{Chloride and the WNK Protein Kinases}

In recent years, there has been mounting evidence on a family of serine/threonine protein kinases, with-no-lysine $(\mathrm{K})(\mathrm{WNK})$ which play a critical role in chloride sensing, intracellular signaling, and regulation. In particular, WNKs serve as an intracellular chloride sensor; it has been shown that autophosphorylation and activation of the kinase take place in response to reduced chloride levels (shown in Fig. 1) [8]. X-ray crystallography studies on WNKs showed the presence of a chloride ion bound directly on the kinase, which established its role as a chloride-sensing protein [9]. Low intracellular chloride activates cation-chloride cotransporters such as NKCC in the thick ascending limb and sodium-chloride cotransporter (NCC) in the distal convoluted tubule through WNKs. NKCC and NCC mediate $\mathrm{Na}+, \mathrm{K}+$, and $\mathrm{Cl}-$ influx which protects the cell against perturbations in osmotic balance. In advanced HF, hypochloremia is commonly seen and is associated with lower diuretic response and less decongestion [10]. A proposed mechanism for this is the action of chloride on WNKs resulting in the upregulation of the NKCC and NCC receptors which leads to increased electrolyte and fluid reabsorption $[9,10]$. The greater amount of NKCC and NCC receptors also has a negative impact on the magnitude of the effect of diuretic therapy. Loop diuretics and thiazide diuretics, 2 first-line therapies for HF, inhibit the NKCC and NCC receptors, respectively. An increase in these receptors may be the underlying mechanism for the diminished efficacy of these medications that was observed in a number of clinical studies [8-10].

\section{The Genomics of Chloride Channel}

With the advancements in pharmacogenomics and personalized medicine, there is increasing interest in finding gene targets for HF treatment. Recent discovery of a gene which encodes a voltage-sensitive chloride
88

Cardiorenal Med 2021;11:87-98 DOI: $10.1159 / 000515604$
Rivera/Alfonso/Golbin/Lo/Lerma/ Volgman/Kazory 


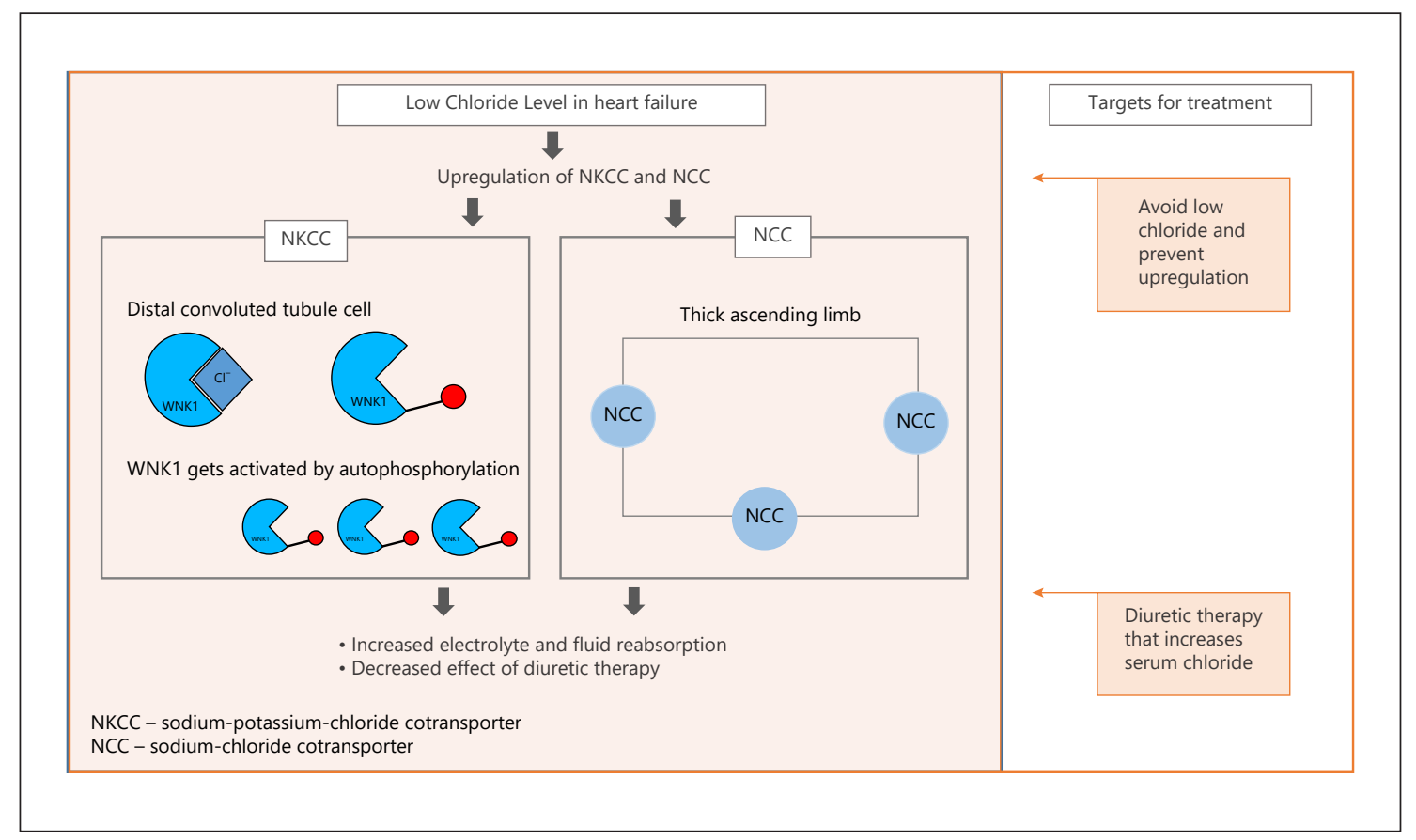

Fig. 1. WNK receptors located in distal convoluted tubule cells play a critical role in chloride sensing, intracellular signaling, and regulation. WNKs serve as an intracellular chloride sensor; when there is normal serum chloride, the WNKK1 is inactivated. During low chloride rates in HF, autophosphorylation and activation of the kinase occur in response to reduced chloride levels. WNK, with-no-lysine (K); HF, heart failure; NKCC, sodiumpotassium-chloride cotransporter; NCC, sodium-chloride cotransporter.

channel (CLCNKA) is potentially associated with renal sodium absorption and salt sensitive hypertension [11]. These potentially linked genetic polymorphisms might actually serve as a marker of pathologic variants of chloride channels that can have implications in HF and hence the cardiorenal axis [11].

In relation to the above findings, further studies eventually found that the potential association with HF of the above gene locus in chromosome 1p36 in HSPB7 was actually brought about in part by a polymorphism of the chloride channel gene CLCNKA itself where a glycine was substituted for the normal arginine at position 83 [11]. In those with the Gly83 variant allele, it was found that there was almost a $50 \%$ decrease in current amplitude and function of the renal chloride channel. The investigators hypothesized that individuals who carry one or more alleles encoding the variant Gly83 variant channel may develop a similar profile to a Bartter-like syndrome of hyperreninemia, which is in turn implicated in the pathogenesis of HF and cardiorenal syndrome. This also predisposes them to a higher risk of HF after a "second hit" in the form of a myocardial infarction and volume overload since the neurohormonal mechanisms are already pathologically activated to begin with [11]. A limitation of this elegant study is the population was homogeneously white, so the effect of these genes for other race or ethnic groups needs further investigation [11].

\section{Chloride and Fluid Retention in HF}

While serum sodium is known to be a major prognosticating factor in HF, recent studies have looked more closely into its sister electrolyte, chloride. In 2015, a landmark study by Grodin et al. [6] raised the possibility that chloride may be just as, if not more, closely associated with adverse outcomes in these patients. Hypochloremia in HF patients is thought to be related to either hemodilution via the mechanisms mentioned in the previous sections, in which case it is associated with a concomitant decrease in sodium, hemoglobin, and hematocrit, or electrolyte depletion such as in diuretic-induced contraction alkalosis. 
Chloride has established actions on the macula densa and the RAAS. A derangement in the chloride levels may contribute further to the dysfunction of the RAAS axis in HF. In fact, as early as 1960, chloride supplementation was already known to be helpful as an adjunct treatment in diuretic-resistant fluid retention states [12]. It was postulated that the induction of a hyperchloremic acidotic state was necessary to make these patients responsive to diuretic therapy. However, as mentioned above, studies that are more recent have found that chloride plays a role in the regulation of chloride transporters (i.e., NKCC and NCC). Hypochloremia leads to an increase in these receptors, resulting in greater electrolyte and fluid reabsorption [13]. This receptor upregulation coupled with the pathologic hyperactivity of RAAS is thought to contribute to the poor prognosis of these patients.

\section{Interaction of the Heart and Kidneys in HF}

While the etiology and mechanistic processes underlying HF may be numerous, central in its pathophysiology is a disturbance in the ability of the ventricles to either pump (HF with reduced ejection fraction) or receive blood (HF with preserved ejection fraction) blood. This dysfunction results in impaired perfusion and oxygen delivery to the peripheral tissues. In compensation, various homeostatic mechanisms are activated, particularly the RAAS, arginine vasopressin system, and the SNS [14]. In the initial stages of $\mathrm{HF}$, perfusion is maintained through vasoconstriction mediated by the SNS. However, the maladaptive RAAS activation will lead to expansion of the blood volume through progressive sodium and water retention. Following this increase in intravascular volume, an increase in hydrostatic pressure within the vessels will tilt the balance in favor of fluid accumulation in the interstitium. Continued increase in the circulating volume then becomes pathologic and results in the clinical signs and symptoms of congestion. In chronic HF, the interstitium can contain several liters of fluid, accounting for the gradual and usually prolonged progression of symptoms. In these patients, a precarious balance exists between the increased perfusion and significant hemodynamic overload [14].

The intrinsic link between cardiac and renal function cannot be overstated. The kidneys serve as one of the first lines of defense countering these mechanisms. However, when the renal blood flow falls below the limits of autoregulation, due to low systolic volume and blood pressure, the glomerular filtration rate (GFR) begins to decrease. Several pathophysiologic pathways have been pos- tulated to contribute to the decline in renal function in this setting. A reduction in systolic blood pressure during admission for acute decompensated heart failure (ADHF) has been found to be significantly associated with worsening renal function [15]. This finding is independent of the cardiac output of the patient due to the unique configuration of the kidney's blood flow, wherein it is more dependent on the pressure rather than the flow or cardiac output. The reduction in systolic pressure also affects the renal baroreceptors, leading to a downstream increase in the RAAS activation further compounding the pathologic changes in circulating volume.

Aside from the arterial component (i.e., low forward flow), venous congestion is also known to be associated with worsening renal function. Damman et al. [16] found that an elevated right atrial pressure, independent of renal blood flow, was associated with a decrease in the GFR. The increased venous pressure translates to increased renal interstitial pressure (i.e., high backward pressure) and therefore interstitial hypoxia. The increased renal interstitial pressure may also compress the renal tubules leading to increased hydrostatic pressure of the Bowman's capsule, altering the Starling forces in favor of a reduction in the GFR [17]. With overfilling of the right ventricle, there is a subsequent decrease in left ventricular filling further compromising the output [17]. An increase in SNS activity modifies the renal filtration coefficient and independently causes systemic vasoconstriction, which contributes to the reduction of GFR. Likely, it is the interplay between these various factors that contribute to the full picture of cardiorenal syndrome. Figure 2 is a summary of pathophysiologic interactions in HF and renal dysfunction.

\section{Clinical Trials - Chloride in Chronic HF}

The study that instigated interest in the role of serum chloride in HF was the post hoc analysis of the BetaBlocker Evaluation of Survival (BEST) trial [18] by Testani et al. [19]. In the univariate model, both serum sodium and serum chloride were strongly associated with mortality, whereas the bicarbonate level was not. Interestingly, only serum chloride remained strongly associated with mortality in the multivariate model. The investigators found that hypochloremia, in the absence of hyponatremia, was a negative prognostic indicator in patients with $\mathrm{HF}$ [19]. In this study, hypochloremia was also found to be associated with a greater loop diuretic dose and higher serum bicarbonate.
Rivera/Alfonso/Golbin/Lo/Lerma/ Volgman/Kazory 


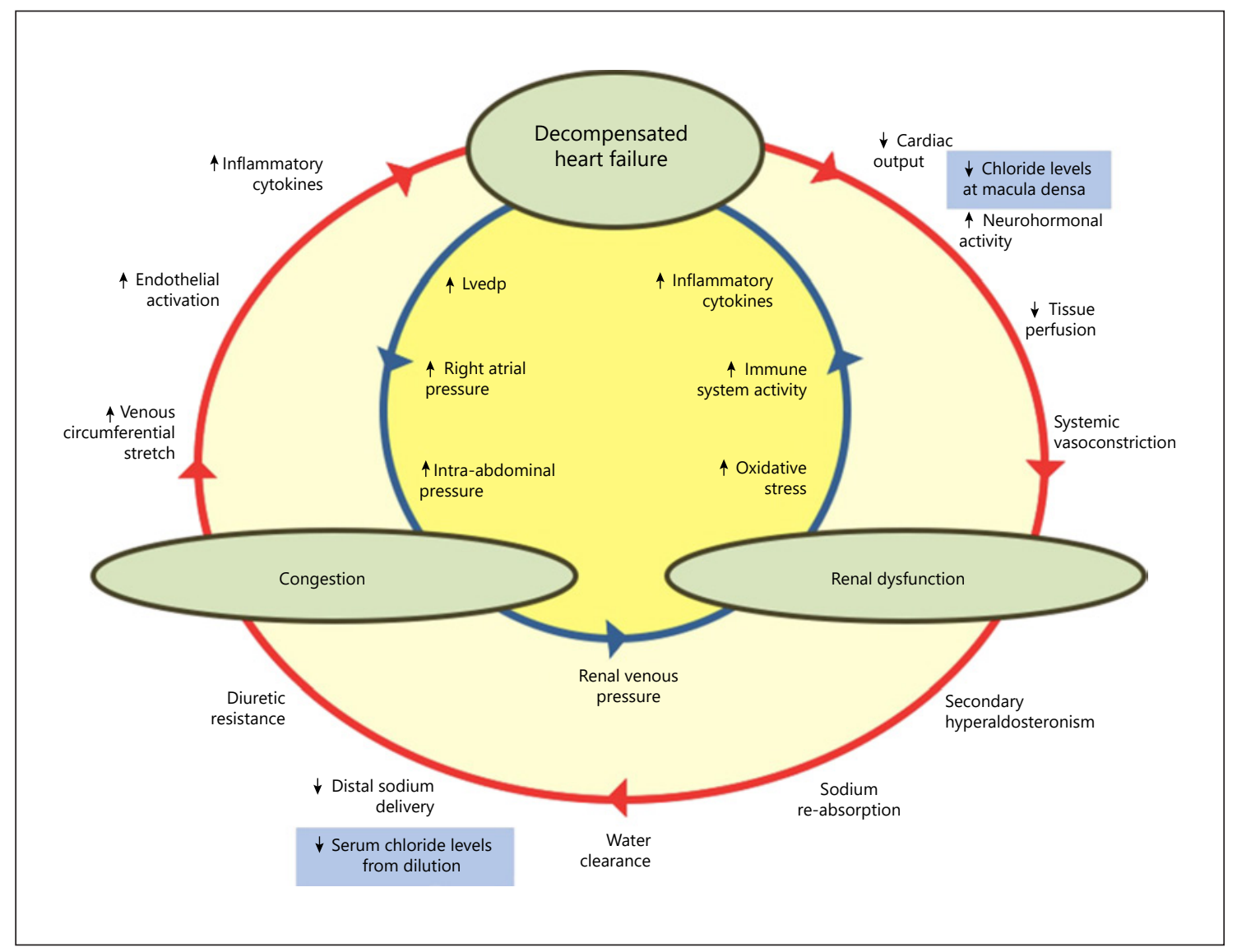

Fig. 2. Bidirectional pathways linking HF, renal dysfunction, and congestion in cardiorenal syndrome. Decompensation of HF can lead to deterioration in renal function via exacerbated neurohormonal activity (i.e., low forward flow) or through fluid overload and renal venous congestion (i.e., high backward pressure). HF, heart failure; LVEDP, left ventricular end-diastolic pressure. Adapted with permission from Kazory A. Clin J Am Soc Nephrol 2016;11:1463-71.

Similar findings were seen in the study by Grodin et al. [20]. They found that every standard deviation decrease in chloride levels $(4.1 \mathrm{mEq} / \mathrm{L})$ at admission to the hospital was associated with a $32 \%$ increase in 5 -year mortality risk even after multivariable adjustment for other factors including serum sodium, cardiorenal biomarkers, selfreported functional status, and medication use. It was noted that serum chloride levels had strong associations with functional status, sodium, BUN, and loop diuretic use, but did not correlate with more traditional markers of HF severity such as left ventricular ejection fraction and B-type natriuretic peptide. Notable in this study is that subgroup analysis revealed that loop diuretic use significantly decreased the association of chloride and mortality. Similar to the study by Testani et al. [19], this implies that the use of loop diuretics contributes significantly to chloride depletion.
The prospective cohort study by Cuthbert et al. [21] showed congruent findings. Low serum chloride was strongly and independently associated with increasing mortality and all-cause mortality or HF hospitalization. The prognosis of patients with hypochloremia, whether from dilution or depletion associated with diuretics, was similar. Grodin et al. [22] analyzed data from the TOPCAT trial which studied patients with HF with preserved ejection fraction. Results were similar to previous studies in that lower serum chloride was associated with an increased risk for cardiovascular death, allcause death, and HF hospitalizations. Hypochloremia was also associated with loop and thiazide diuretic use which implicates these medications in chronic chloride depletion.

However, different disease states and patient population may affect these results. Ferreira et al. [23] analyzed 


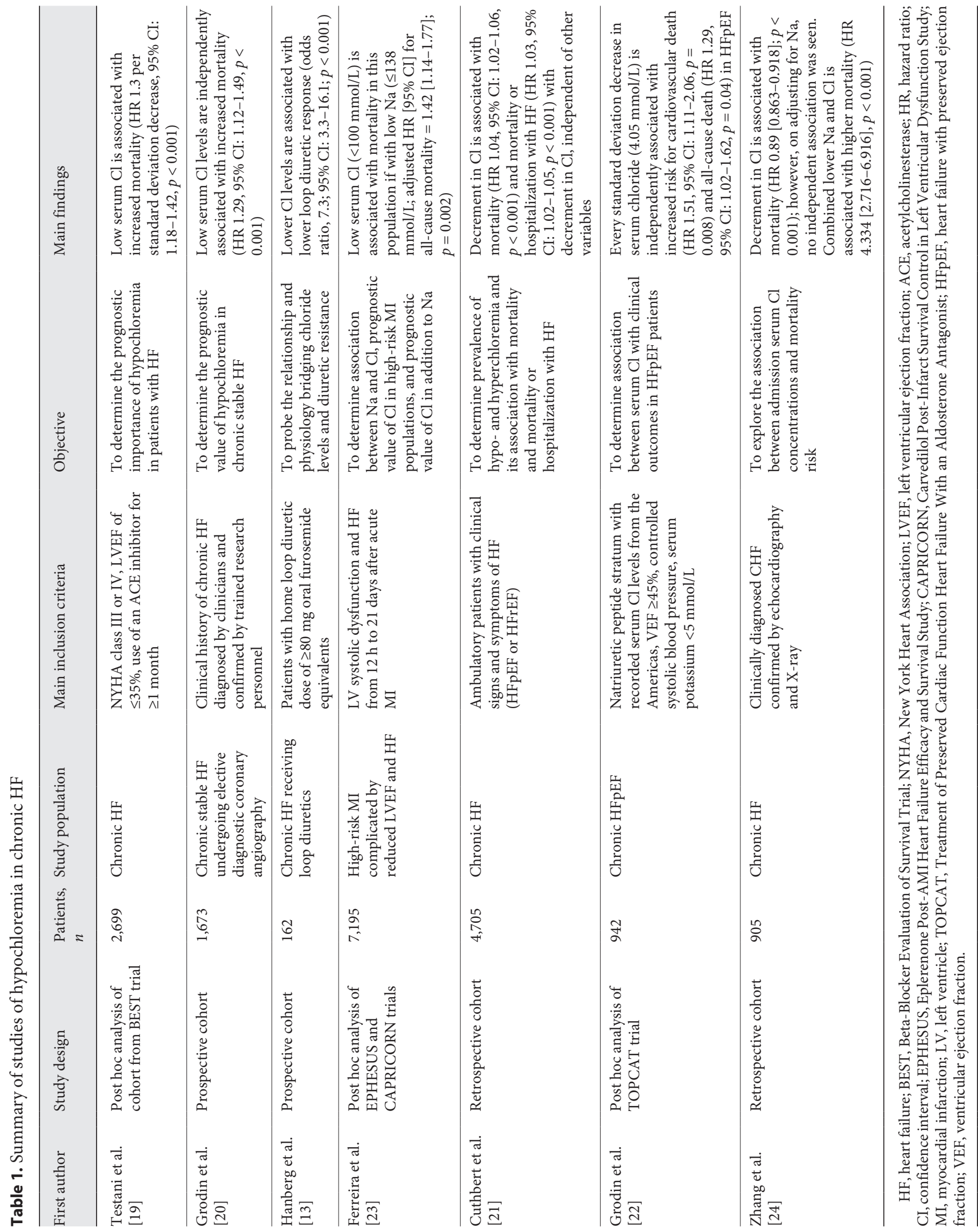




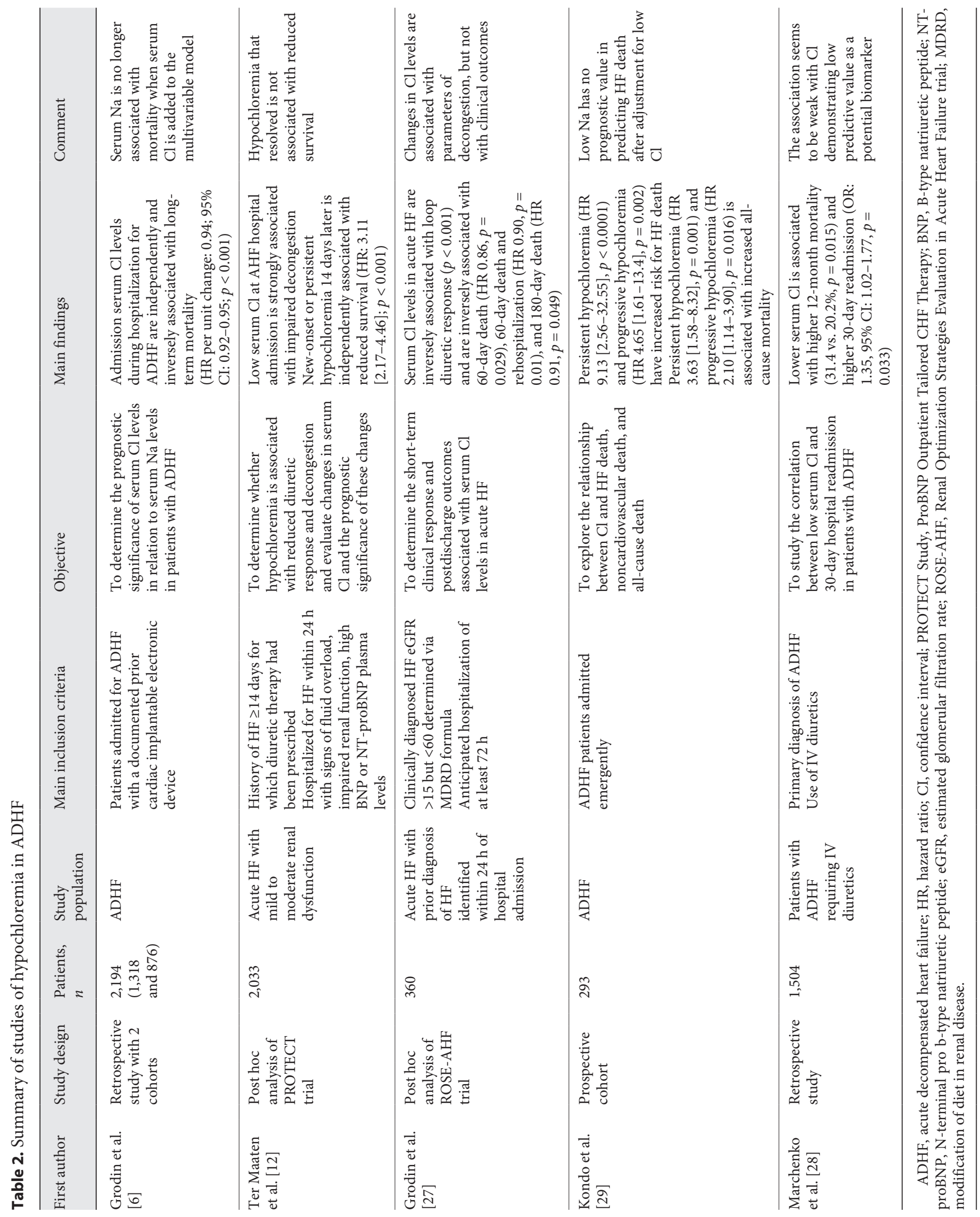


the prognostic value and association of serum chloride with adverse outcomes in subjects with acute myocardial infarction complicated by reduced left ventricular function and HF. Serum chloride was independently associated with all-cause mortality and cardiovascular mortality but only in patients with the lower serum sodium tertile $(\mathrm{Na}<138 \mathrm{mmol} / \mathrm{L})$. Zhang et al. [24] studied the prognostic value of serum chloride in a Chinese Han population in China. The study found that each unitary decrease in chloride was associated with an $11 \%$ increase in risk of mortality. In both of these studies, the association between mortality and hypochloremia was independent of the use of loop diuretics.

Two studies on the association of hypochloremia and mortality were presented at the American College of Cardiology in March 2020. One study found that hypochloremia was a strong independent predictor of mortality and that an abnormal serum chloride on admission is associated with increased short- and long-term mortality [25]. A study of 289 patients undergoing left ventricular assist device placement found that hypochloremia was associated with decreased survival at 1 year [26].

There seems to be a strong association among serum sodium, serum chloride, and mortality; however, as seen above, the precise relationship and the potential confounding elements still remain elusive. Table 1 is a summary of clinical trials for patients with chronic HF.

\section{Clinical Trials - Chloride in Acute HF}

The first landmark study that looked at the role of chloride in ADHF was the retrospective cohort study of Grodin et al. [6]. The study was composed of patients with a history of chronic HF and cardiac implantable electronic device who were admitted for ADHF. Interestingly, serum sodium levels did not correlate with increased mortality after multivariate adjustment. Instead, the authors reported that lower levels of chloride on admission and hypochloremia on discharge were associated with increased all-cause mortality, independent of sodium levels. Similar findings were observed in a post hoc analysis of the data from the ROSE-AHF (Renal Optimization Strategies Evaluation in Acute Heart Failure) trial. The authors found that baseline chloride levels did not correlate with worsening or persistent HF but had a negative association with response to diuretics and diuretic efficiency, increased 60-day and 180-day mortality, and rehospitalization rate [27]. The use of diuretics lowered serum chloride levels during admission. However, this acute change in chloride was not associated with increased mortality. Adjustment for prehospital diuretic use also decreased the association of baseline chloride levels and mortality. These findings imply that while loop diuretics can deplete chloride, its impact on mortality is less pronounced compared with other causes of hypochloremia in HF (i.e., dilution).

Further investigation on the association between hypochloremia and rehospitalization was done by Marchenko et al. [28]. In their study, hypochloremia on either admission or discharge was found to be independently associated with a greater 30 -day readmission rate. However, the predictive value of chloride for readmission was found to be low. Mortality at 12 months was also observed to increase in this setting. While the study grouped those with hypochloremia on either admission or discharge as one during the analysis, it is notable that the number of patients with hypochloremia at discharge was greater than those on admission and that only a small portion of the patients were hypochloremic from admission to discharge. This change likely reflects the effect of decongestive therapy during admission. Hence, the magnitude of the effect of therapy-related hypochloremia on prognosis is unknown in this study.

The value of baseline chloride as a reliable prognostic marker was challenged by Ter Maaten et al. [12]. In their analysis of a cohort from the PROTECT (Placebo-Controlled Randomized Study of the Selective A1 Adenosine Receptor Antagonist Rolofylline for Patients Hospitalized with Acute Decompensated Heart Failure and Volume Overload to Assess Treatment Effect on Congestion and Renal Function) trial, they found that baseline serum chloride was not significantly associated with mortality at 180 days when adjusted for baseline sodium. Instead, patients with persistent hypochloremia until day 14 and patients with new-onset hypochloremia at day 14 had the strongest association with mortality [12]. This study raises the possibility that treatment-related hypochloremia may lead to worse outcomes. The association with serial changes in chloride and mortality was also seen by Kondo et al. [29]. Those patients with persistent hypochloremia and progressive hypochloremia were at increased risk of mortality due to progressive HF. Of note, the dose of loop diuretics and thiazides during hospitalization was associated with progressive hypochloremia and therefore with poorer outcomes. Table 2 is a summary of clinical trials for patients with acute HF.
Cardiorenal Med 2021;11:87-98 DOI: 10.1159/000515604
Rivera/Alfonso/Golbin/Lo/Lerma/ Volgman/Kazory 
Table 3. Common diuretics used in HF and its effects on chloride

\begin{tabular}{|c|c|c|}
\hline Diuretic & $\begin{array}{l}\text { Typical maintenance dose, } \\
\text { mg PO (maximum dose, mg, IV) }\end{array}$ & Effect on serum chloride \\
\hline \multicolumn{3}{|l|}{ Loop diuretics [35] } \\
\hline Furosemide & $40-240(600)$ & \multirow{3}{*}{$\begin{array}{l}\text { Decreases serum chloride by preventing reabsorption in the } \\
\text { ascending loop of Henle }\end{array}$} \\
\hline Bumetanide & $1-5(10)$ & \\
\hline Torsemide & $10-20(200)$ & \\
\hline \multicolumn{3}{|c|}{ Thiazide and thiazide-like diuretics [36] } \\
\hline HCTZ & $12.5-100(200)$ & \multirow{4}{*}{$\begin{array}{l}\text { Decreases serum chloride by blocking electroneutral } \mathrm{NaCl} \\
\text { absorption at the distal convoluted tubule, connecting tubule, } \\
\text { and early collecting duct [32] }\end{array}$} \\
\hline Metolazone & $2.5-10(20)$ & \\
\hline Chlorthalidone & $25-200(100)$ & \\
\hline Chlorothiazide & $500-100(1000)$ & \\
\hline \multicolumn{3}{|c|}{ Mineralocorticoid receptor antagonists [37] } \\
\hline Spironolactone & $25-50(50-100)$ & \multirow[t]{2}{*}{ No significant effect [38] } \\
\hline Eplerenone & $25(50)$ & \\
\hline \multicolumn{3}{|c|}{ Vasopressin antagonist } \\
\hline Tolvaptan & $30(60)$ & No significant effect \\
\hline \multicolumn{3}{|l|}{ Other mechanisms } \\
\hline \multirow[t]{4}{*}{ Acetazolamide [32] } & \multirow[t]{4}{*}{250 (500 mg $3 \times /$ day $)$} & A potential chloride-regaining diuretic [32] \\
\hline & & Possesses a "non-reabsorbable anion-like effect" \\
\hline & & Brings about excretion of bicarbonate into the urinary tubules \\
\hline & & $\begin{array}{l}\text { with interchangeable absorption of filtrated chloride into the } \\
\text { blood and concurrent excretion of potassium into the urine [32] }\end{array}$ \\
\hline
\end{tabular}

HF, heart failure; mg, milligrams; PO, per orem; IV, intravenous; HCTZ, hydrochlorothiazide; NaCl, sodium chloride.

\section{Common Diuretics Used in HF}

Based on the latest position statement of the European Society of Cardiology on the use of diuretics in HF with congestion, the use of loop diuretics is still the first-line treatment of congestion followed by thiazide and acetazolamide if the desired urine output is not achieved. Loop and thiazide diuretics can potentially deplete serum chloride primarily by preventing its reabsorption [3032]. On the other hand, acetazolamide which has been considered as a potential chloride-regaining diuretic increases serum chloride levels, independent of sodium, through inhibition of intracellular and luminal carbonic anhydrase in the proximal tubules $[33,34]$. Other diuretics used in HF like spironolactone and tolvaptan has no significant effect on the serum chloride. Table 3 is a summary of common diuretics used in HF and their effects on serum chloride.

\section{Serum Chloride as a Treatment Target}

Given the aforementioned findings, several studies have attempted to investigate the therapeutic impact of chloride supplementation. The isolated increase in serum chloride can be achieved either by supplementation of sodium-free chloride or by administration of an agent or medication that increases serum chloride without affecting serum sodium (i.e., acetazolamide). As early as the 1960s, an interventional trial by Rubin et al. [35] studied the effect of sodium-free chloride (i.e., lysine monohydrochloride) supplementation on patients with refractory congestion. The results showed that it enhanced fluid and electrolyte excretion in patients previously resistant to diuretics. Based on that concept, Hanberg et al. [13] performed their experiment on 10 patients (pilot study) which had inconsistent results; instead of reducing renin level, it increased it. However, the metrics of decongestion were improved implying increased efficiency of the diuretics. There is an ongoing randomized controlled trial [36] on the effect of lysine chloride supplementation in acute and chronic HF patients on the change in blood volume and other endpoints. 
Fig. 3. Important findings of the clinical trials looking at the role of serum chloride among patients with acute and chronic HF. Several studies have shown significant association of hypochloremia with mortality with varying results depending on disease states and study population. Intracellular chloride sensors play a role in diuretic resistance, and these sensors may be treatment targets. An amino acid substitution in the CLCNKA gene affects the renal chloride channels and is associated with increase in HF regardless of myocardial damage. HF, heart failure.

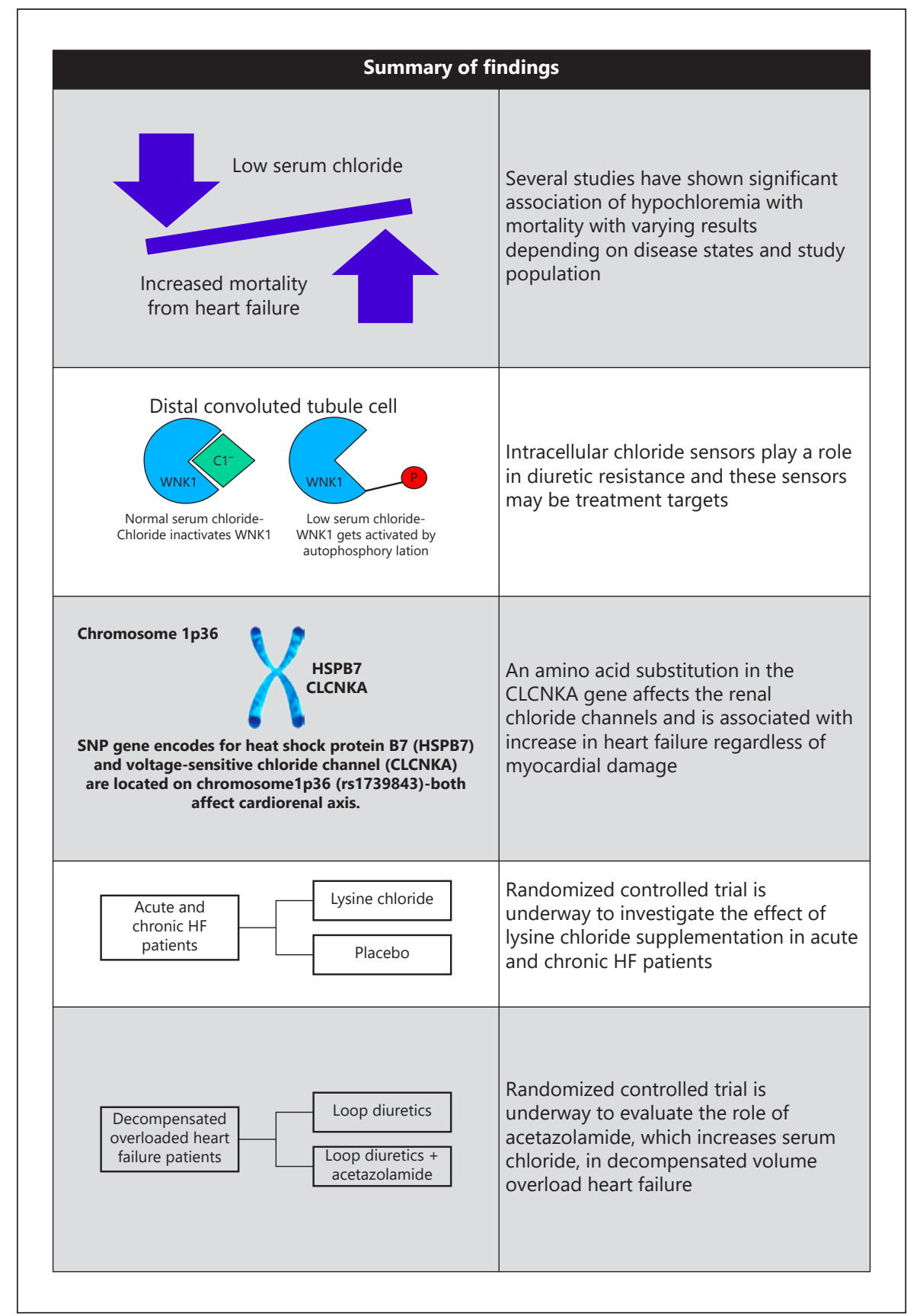

The impact of acetazolamide administration has also been investigated by several studies. Kataoka et al. [37] used acetazolamide in patients with ADHF and chronic HF to determine if its use can increase and sustain chloride levels. The study found that chloride concentrations improved within 10 days and were sustained for at least 60 days. On the other hand, a randomized controlled trial by Verbrugge et al. [38] looked at acetazolamide's effect on improving natriuresis in HF. Acetazolamide was added to low-dose loop diuretics in the treatment arm, which showed a similar impact on urine sodium excretion and metrics of decongestion that high-dose loop diuretics had (i.e., it increased their efficiency). Another ongoing larger clinical trial, ADVOR (Acetazolamide in Decompensated Heart Failure with Volume Overload) [39], will use the same hypothesis to determine whether adding acetazol- 
amide will improve decongestion when combined with loop diuretics in ADHF, potentially leading to improved clinical outcomes.

\section{Conclusion}

Because the macula densa relies on chloride levels in sensing salt load and volume status, chloride plays a significant role in fluid homeostasis in HF. Also, by serving as an intracellular chloride sensor, the WNK pathway probably plays a role in diuretic resistance. Genetic studies have shown that in a Caucasian population, an amino acid substitution in the CLCNKA gene affects the renal chloride channels and is associated with increase in HF regardless of myocardial damage. While several studies in both acute and chronic HF populations have demonstrated the prognostic value of hypochloremia, interventional clinical trials that explored serum chloride as a therapeutic target have so far been inconclusive. Prospective randomized controlled studies are underway which may elucidate the role of serum chloride as a therapeutic target to improve outcomes in patients with HF. These studies should shed light on whether serum chloride should be integrated into current models for prediction of HF prognosis. Figure 3 is a summary of the findings of this review.

\section{Acknowledgements}

Figures 1 and 3 were created by Caroline Volgman, Administrative Project Assistant, Community Health Equity and Engagement, Rush Health Systems Management. Special thanks to Dr. John C. Añonuevo (PGH Internal Medicine Department Chair) for his support and guidance.

\section{Conflict of Interest Statement}

A.K. has the following potential conflicts of interest: Baxter, Inc. (Cardiology Advisory Board and consultancy fee), CHF Solutions, Inc. (Medical Advisory Board and consultancy fee), and W.L. Gore Inc. (consultancy fee). A.S.V. has no conflicts of interest. A.S.V. - MSD/Bayer Virtual Global Advisory Board Member, Bristol Myers Squibb Foundation Diverse Clinical Investigator Career Development Program (DCICDP), National Advisory Committee (NAC), NIH Clinical Trials, and Apple Inc., stock.

\section{Funding Sources}

No specific financial support was obtained for preparation of this article.

\section{Author Contributions}

Frederick Rivera, Pia Alfonso, and Jem Marie Golbin made the first draft. Additional inputs on the renal physiology and cardiorenal interaction were added by Dr. Kozary, Dr. Lo, and Dr. Lerma. The manuscript was edited by Dr. Volgman and worked with Caroline Volgman to create Figures 1 and 3.

\section{References}

1 Braunwald E. The war against heart failure: the Lancet lecture. Lancet. 2015 Feb 28; 385(9970):812-24.

2 Savarese G, Lund LH. Global public health burden of heart failure. Card Fail Rev. 2017; 3(1):7-11.

3 Waikar SS, Mount DB, Curhan GC. Mortality after hospitalization with mild, moderate, and severe hyponatremia. Am J Med. 2009 Sep; 122(9):857-65.

4 Berend K, van Hulsteijn LH, Gans RO. Chloride: the queen of electrolytes? Eur J Intern Med. 2012 Apr 1;23(3):203-11.

5 Cox ZL, Stevenson LW. The weight of evidence for diuretics and parachutes. J Am Coll Cardiol. 2020;76(6):680-3.

6 Grodin JL, Simon J, Hachamovitch R, Wu Y, Jackson G, Halkar M, et al. Prognostic role of serum chloride levels in acute decompensated heart failure. J Am Coll Cardiol. 2015 Aug; 66(6):659-66.

7 Powers F. The role of chloride in acid-base balance. J Intraven Nurs. 1999;22(5):286-91.
8 Moriguchi T, Urushiyama S, Hisamoto N, Iemura S, Uchida S, Natsume T, et al. WNK1 regulates phosphorylation of cation-chloridecoupled cotransporters via the STE20-related kinases, SPAK and OSR1. J Biol Chem. 2005 Dec 30;280(52):42685-93.

9 Piala AT, Moon TM, Akella R, He H, Cobb $\mathrm{MH}$, Goldsmith EJ. Chloride sensing by WNK1 involves inhibition of autophosphorylation. Sci Signal. 2014 May 6;7(324):ra41.

10 Ponce-Coria J, San-Cristobal P, Kahle KT, Vazquez N, Pacheco-Alvarez D, de los Heros $\mathrm{P}$, et al. Regulation of NKCC2 by a chloridesensing mechanism involving the WNK3 and SPAK kinases. Proc Natl Acad Sci USA. 2008 Jun 17;105(24):8458-63.

11 Cappola TP, Matkovich SJ, Wang W, van Booven D, Li M, Wang X, et al. Loss-of-function DNA sequence variant in the CLCNKA chloride channel implicates the cardio-renal axis in interindividual heart failure risk variation. Proc Natl Acad Sci USA. 2011 Feb 8; 108(6):2456-61.
12 Ter Maaten JM, Damman K, Hanberg JS, Givertz MM, Metra M, O'Connor CM, et al. Hypochloremia, diuretic resistance, and outcome in patients with acute heart failure. Circ Heart Fail. 2016;9(8):e003109.

13 Hanberg JS, Rao V, Ter Maaten JM, Laur O, Brisco MA, Perry Wilson F, et al. Hypochloremia and diuretic resistance in heart failure: mechanistic insights. Circ Heart Fail. 2016; 9(8):e003180.

14 Testani JM, Coca SG, McCauley BD, Shannon $\mathrm{RP}$, Kimmel SE. Impact of changes in blood pressure during the treatment of acute decompensated heart failure on renal and clinical outcomes. Eur J Heart Fail. 2011 Aug; 13(8):877-84.

15 Miller WL. Fluid Volume Overload and Congestion in Heart Failure. Circulation. 2016 Aug. Available from:https://www.ahajournals.org/doi/abs/10.1161/CIRCHEARTFAILURE.115.002922. 
16 Damman K, Navis G, Smilde TD, Voors AA, van der Bij W, van Veldhuisen DJ, et al. Decreased cardiac output, venous congestion and the association with renal impairment in patients with cardiac dysfunction. Eur J Heart Fail. 2007;9(9):872-8.

17 Alpert JS. The effect of right ventricular dysfunction on left ventricular form and function. Chest. 2001 Jun 1;119(6):1632-3.

18 Beta-Blocker Evaluation of Survival Trial Investigators; Eichhorn EJ, Eichhorn EJ, Domanski MJ, Krause-Steinrauf H, Bristow MR, et al. A trial of the beta-blocker bucindolol in patients with advanced chronic heart failure. N Engl J Med. 2001 May 31;344(22): 1659-67.

19 Testani JM, Hanberg JS, Arroyo JP, Brisco MA, Ter Maaten JM, Wilson FP, et al. Hypochloraemia is strongly and independently associated with mortality in patients with chronic heart failure. Eur J Heart Fail. 2016; 18(6):660-8.

20 Grodin JL, Verbrugge FH, Ellis SG, Mullens W, Testani JM, Tang WH. Importance of abnormal chloride homeostasis in stable chronic heart failure. Circ Heart Fail. 2016 Jan;9(1): e002453.

21 Cuthbert JJ, Pellicori P, Rigby A, Pan D, Kazmi S, Shah P, et al. Low serum chloride in patients with chronic heart failure: clinical associations and prognostic significance: chloride in chronic heart failure. Eur J Heart Fail. 2018 Oct;20(10):1426-35.

22 Grodin JL, Testani JM, Pandey A, Sambandam K, Drazner MH, Fang JC, et al. Perturbations in serum chloride homeostasis in heart failure with preserved ejection fraction: insights from TOPCAT. Eur J Heart Fail. 2018; 20(10):1436-43.

23 Ferreira JP, Girerd N, Duarte K, Coiro S, McMurray JJ, Dargie HJ, et al. Serum chloride and sodium interplay in patients with acute myocardial infarction and heart failure with reduced ejection fraction: an analysis from the high-risk myocardial infarction database initiative. Circe Heart Fail. 2017;10(2): e003500.

24 Zhang Y, Peng R, Li X, Yu J, Chen X, Zhou Z. Serum chloride as a novel marker for adding prognostic information of mortality in chronic heart failure. Clin Chim Acta. 2018 Aug; 483:112-8.

25 Breen T, Brueske B, Sidhu MS, Murphree DH, Kashani KB, Barsness GW, et al. Abnormal serum sodium is associated with increased mortality among unselected cardiac intensive care unit patients. J Am Heart Assoc. 2020; 9(2):e014140.

26 Trovato V, Singh H, Smith SA. Hypochloremia At Time Of Left Ventricular Assist Device Implantation Is Associated With Decreased One Year Survival. J Am Coll Cardiol. 2020; 75(11):905.

27 Grodin JL, Sun JL, Anstrom KJ, Chen HH, Starling RC, Testani JM, et al. Implications of serum chloride homeostasis in acute heart failure (from ROSE-AHF). Am J Cardiol. 2017 Jan;119(1):78-83.

28 Marchenko R, Sigal A, Wasser TE, Reyer J, Green J, Mercogliano C, et al. Hypochloraemia and 30 day readmission rate in patients with acute decompensated heart failure. ESC Heart Fail. 2020 Jun;7(3):903-7.

29 Kondo T, Yamada T, Tamaki S, Morita T, Furukawa $\mathrm{Y}$, Iwasaki $\mathrm{Y}$, et al. Serial change in serum chloride during hospitalization could predict heart failure death in acute decompensated heart failure patients. Circ J. 2018; 82(4):1041-50.

30 Felker GM, Ellison DH, Mullens W, Cox ZL, Testani JM. Diuretic therapy for patients with heart failure: JACC state-of-the-art review. J Am Coll Cardiol. 2020;75(10):1178-95.

31 Mullens W, Damman K, Harjola VP, Mebazaa A, Brunner-La Rocca HP, Martens P, et al. The use of diuretics in heart failure with con- gestion - a position statement from the heart failure association of the European society of cardiology. Eur J Heart Fail. 2019;21(2):13755.

32 Kazory A, Ronco C. Emergence of chloride as an overlooked cardiorenal connector in heart failure. Blood Purif. 2020;49(1-2):219-21.

33 Brater DC. Pharmacology of diuretics. Am J Med Sci. 2000;319(1):38-50.

34 Dyckner T, Wester PO, Widman L. Effects of spironolactone on serum and muscle electrolytes in patients on long-term diuretic therapy for congestive heart failure and/or arterial hypertension. Eur J Clin Pharmacol. 1986;30(5): 535-40.

35 Rubin AL, Spritz N, Mead AW, Herrmann RA, Braveman WS, Luckey EH. The use of Llysine monomydrochloride in combination with mercurial diuretics in the treatment of refractory fluid retention. Circulation. 1960 Mar;21(3):332-6.

36 Mechanism and Effects of Manipulating Chloride Homeostasis in Acute Heart Failure - Full Text View - ClinicalTrials.gov [Internet]. [cited 2020 Dec 7]. Available from: https: //clinicaltrials.gov/ct2/show/NCT03446651

37 Kataoka H. Acetazolamide as a potent chloride-regaining diuretic: short- and long-term effects, and its pharmacologic role under the "chloride theory" for heart failure pathophysiology. Heart Vessels. 2019 Dec;34(12):195260.

38 Verbrugge FH, Martens P, Ameloot K, Haemels V, Penders J, Dupont M, et al. Acetazolamide to increase natriuresis in congestive heart failure at high risk for diuretic resistance. Eur J Heart Fail. 2019;21(11):1415-22.

39 Mullens W. A multi-center, randomized, double-blind, phase iv clinical trial on the diuretic effects of acetazolamide in patients with decompensated heart failure and volume overload. 2020 Jun. Available from: https:// clinicaltrials.gov/ct2/show/NCT03505788 